\title{
MIND-BODY INTEGRATION IN DANCE MOVEMENT THERAPY
}

\author{
Hongju $\mathrm{Li}^{1}$, \& Xindi $\mathrm{Cao}^{2}$ \\ ${ }^{1}$ School of Arts and Communication, Beijing Normal University (China) \\ ${ }^{2}$ School of Philosophy, Beijing Normal University (China)
}

\begin{abstract}
Mind-body integration is a key element for a successful dance movement therapy (DMT). As the connection between mind and body is reconnected, the clients can not only express but also improve their mental state through body movement. The integration of mind and body can be viewed from to aspects, namely the first-person experience and the third-person phenomenon, both of which play a crucial role in the therapeutic process of DMT. The first-person experience transcends the mind's neurobiological phenomenon, which is relatively more important for the clients, while the third-person observation is based on the body's reflection of mind, often adopted by the therapists. The relationship between mind and body can be validated by the mirror neuron theory, which is one of the theoretical proofs and inspirations of DMT. Unlike the notion of mind-body differentiation in western classical philosophy, ancient Chinese thoughts had not separated them. The Chinese character "shen" is considered as the whole of flesh and soul. The abundant Chinese thoughts concerning mind-body theory can be considered as a suitable route of the exploration of mind-body integration.
\end{abstract}

Keywords: Dance movement therapy, mind-body integration, philosophy of mind, mirror neuron, Chinese philosophy.

\section{The mind-body problem in dance-movement therapy}

As a mental healing method, Dance-Movement Therapy (DMT) may incur censure because its main therapeutic process focuses on the body. Whether the body effect can, going through the blood and flesh, influence the mental state, seems questionable. Once there was barrier for the DMT therapists to find their own position in the psychotherapy institutions and their certification is controversy. (Meekmus,2002 p12-13) Although DMT is getting more and more accepted in the field of psychotherapy, worry and suspicion from the clients and other co-workers still exists. Therefore, rebuilding the connection between mind and body is necessary for the legitimacy and reliability of DMT.

\section{The mind-body theories: From philosophy and psychology perspectives}

\subsection{The first-person ontology in the mind-body integration}

Views of mind-body problem can be categorized into dualism and monism. Generally, dualism regards mind and body as two separate entities, which is widely accepted historically, while monism, solving the dualism's difficulty of the interaction of mind and body, takes either mind or body as the foundation. According to which plays the role of the foundation, Monism can be divided into two types: idealism and materialism. The former takes the mind as the ultimate essence and the body attach to it, while the later takes body as the source of mind. In this essay, we would discuss the mind-body relationship base on materialism for the reason that being compatible with modern psychological science, materialism is not only prevalent in contemporary philosophy of mind, but also a significant theoretical prerequisite of DMT.

From the materialism point of view, mind is the consequence of physical processes, such as neural processes. Opponents may argue that due to this understanding, mind is nothing. In other words, mind does not necessarily exists and every mental process is nothing but physical process. In this way, any psychotherapies, including DMT, ultimately aim at effecting the neural state. However, mind cannot be reduced to physical process. According to Searle's "biological naturalism", which is a variant of materialism, mental state is causally reducible, not ontologically reducible to its neurobiological basis. (Searle,2004 p113) The remarkable difference between mechanical materialism and "biological naturalism" lies in the mind's relative independence. "Biological naturalism" approves the body's effect on mind and at the same time, admit mind's own features. This, on one hand, legitimize DMT as a mental 
healing method through the body movement. On the other hand, it highlights its trait as expressive art therapy, which rely partly on spiritual interaction.

Since the mind cannot be ontologically reduced to the body, there must be something beyond the physical process that is produced by the body, which constitute the mental experience. It is helpful to differentiate the concepts of "first person" and "third person" features. (Searle,2004 p111) Some mental phenomena can be observed and explained from a third-person perspective, which is commonly researched by psychological scientists. These phenomena are directly determined by the neurobiological state. However, what scientific mindset and researches often neglect is the first-person experience. It is the first-person features that make the mind transcend the physical features, while at the same time, they are closely attached to the body state. The "thirsty" example put forward by Searle may well explain these two terms. When you are thirsty, a set of neural activities produce the signal showing that you are thirsty. This is a third-person phenomenon. Apart from that, as a sensible subject, you experience that feeling of thirsty, which is a consequence of your body's lack of water. The feeling of thirsty cannot be exactly and completely described by any word language, including the scientific explanation of neurobiological process. It can only be experienced by the first person. The first-person experience is an indispensable part of mind-body integration, but this mental state is usually separated from the body because of the disregard of body perception.

Therefore, for those psychologically disturbed people who are not able to be aware of their mental state correctly, it is an important step to reestablish the first-person perception. First-person mental state is also a puzzle for people who have experienced body-related trauma, such as sexual abuse and domestic violence concerning physical abuse. They suffer from "detached state" where they alienate their body to avoid recalling the terrible memory. (Meekmus,2002 p77) Under this circumstance, their body and mind are separated. Although this separation enables them to get rid of the trauma for the moment, it may cause severe and long-term damage to their psychological health. DMT, as a therapeutic method that involves both mind and body, is suitable for this case because of its specialty of embodiment. The key point of reconnecting the mind and body is first-person experience. More specifically, through the movement of body, they consciously learn to experience their own feeling of the body under the instruction of the therapist. Apart from the clients, the therapists can also use first person experience during the therapeutic process. For instance, the therapists may sometimes "feel" the clients" physical and mental state as well as the atmosphere of the group. This kind of feeling can be perceptual and emotional instead of rational and normative.

As a common method in psychological therapy, the third-person perspective is also applied in DMT. Observing the client's improvisation, the therapist figure out the mental state through the metaphor of the body movement. Although the client may also get out of their situation to analyze their own mental phenomena from a third-person view, most third-person features are in the hands of the therapist. Therefore, the integration of mind and body in DMT owe to the interaction of first-person and third-person perspective, namely the client and the therapists.

\subsection{The application of mirror neuron theory in dance-movement therapy}

The notion of monism can be validated by the mirror neuron theory. It proves that the practice of a movement can be combined with the perception of the movement. The theory was found by Giacomo Rizzolatti and his group in the University of Parma. They studied the rhesus monkeys' neuron system and found that the ventral premotor cortex of the rhesuses might deliver electrical signal when carrying on a movement, and the similar neural phenomenon occurred when they were observing other rhesuses, or even human, to do it. From this experiment, researchers conclude that motor center of the brain is related to not only body movements, but also the understanding of others' movements. (Ye,Zeng,2013) This can be implied to DMT in various of ways. For instance, therapists may mirror their clients' movement to put themselves into the clients' situation. Through experiencing the clients' movement, the therapists can figure out what is happening in the clients' inner world, namely their mental state. On the other hand, the mirror neuron theory also shows that the act of watching others' movement may have similar effect in the brain process as they actually do it. Though it seems contrast to the consensus of DMT that it is irreplaceable to experience the movement in the flesh, which means truly moving our own body in the space, it may lead to a different type of technique in DMT. For the clients who cannot freely move the body, it is helpful to let them watch the therapist's movement. This method is proved effective in a therapeutic process for children with cerebral palsy. There's much more to explore about this "watching" method of DMT.

\section{The Mind-body Concepts of Chinese Thought}

The wisdom of moderation (zhong yong) and harmony (he) is deeply rooted in Chinese traditional culture, instructing people to keep inner peace and emotional balance. Hence, the passages in Confucian and Taoist classics are more than philosophical thoughts and moral norms. They play a crucial 
role in people's self-adjusting of mind and body. To some extent, Chinese philosophical thoughts, infiltrating into Chinese people's personality and life outlook, can be compared to a "psychotherapist". This kind of mental adjustment through long-term reading, thinking and practicing is more of a first-person experience other than a third-person assistance. So it can be considered as a suitable route of the exploration of mind-body integration.

In Chinese culture, mind and body are radically a whole entity. Unlike the notion of mind-body differentiation in western classical philosophy, ancient Chinese thoughts had not separated them. The Chinese character "shen" is considered as the whole of flesh and soul. Wang Yangming, a philosopher of Song-Ming Neo-Confucianism, stated that " The ears, eyes, mouth, nose and limbs are parts of the body. If it is not the mind, how can they watch, listen, speak and move? The mind also cannot watch, listen, speak and move without ears, eyes, mouth, nose and limbs. Therefore, where there is no mind, there is no body. Where there is no body, there is no mind."(Wang,1992 p90-91)1 ) The body cannot function without the mind and the mind cannot function without the basis of the organs of the body. Relying on each other, the mind and body can only watch, listen, speak and move when they function cooperatively. In spite of that, he also thought that the primitive integrity can be interrupted by the "inner desire" and the only way to regain the integrity is to "sit still" and "cease the contemplation". These two concepts, one applies to the body and the other applies to the mind, are actually two aspects of the one action. The reintegration of mind and body requires mind-body practice as a whole.

Unlike DMT, Wang Yangming's practice only involves first-person experience. It demands intense self-awareness and attention, which only few people own. For the clients of DMT, they need more specific instructions based on the third-person observation of the therapists. However, the ability of first-person self-perception is a necessary element of mind-body integration. Hence, if DMT enhance the clients' ability of self-awareness, they would be able to apply it to their daily life, either during the gap of of after a therapeutic process so that they could keep a balanced mind-body relationship.

\section{Summary}

In this essay, I presented western and eastern thought proving mind-body integration and its significance in DMT practice. In both cases, I attach importance to first-person experience, which is a vital element of DMT, because this is a unique trait of DMT as an art therapy method involving personal sensibility and as a body-based therapy directly connecting the mind and body. These thoughts may inspire the DMT practice towards a more humanized and individualized direction.

\section{References}

Meekums, Bonnie, \& Yu,Zemei translate. (2016). Dance Movement Therapy: A Creative Psychotherapeutic Approach. Chongqing: Chongqing University Press Limited Corporation.

Searle, John R. (2004). Mind: a Brief Introduction. USA: Oxford University Press.

Wu, Guang, \& Qian, Dong, Yao. (1992). Completed Works of Wang Yangming. Shanghai: Shanghai Classics Publishing House.

Ye, Haosheng, \& Zeng, Hong. (2013). The Discovery of Mirror Neurons and the Transcendence over Mind-Body Dualism. Journal of Psychological Science, 2013,36(5), 1230-1236.

${ }^{1}$ Wang Yangming, edited by Wu Guang, Qian Ming, Dong Ping, and Yao Yanfu. Wang Yangming's Complete Works Part 1[M]. Shanghai Ancient Books Publishing House, 1992. Pages 90-91.. 